\section{PET imaging of 18F-florbetapir in cognitively impaired individ- uals: Lack of activity within the cerebellar cortex}

\author{
Michael A. Meyer, Allison Caccia, \\ Danielle Martinez, Mark A. Mingos \\ Departments of Neurology and Nuclear \\ Medicine, Guthrie Clinic, Sayre PA, \\ USA
}

\begin{abstract}
Ten individuals suspected of having possible Alzheimer disease underwent PET imaging using 18F-Flubetapir. Only one of ten individuals had a pattern typical for normal elderly control subjects with 9 of the 10 showing a Alzheimer type pattern for the cerebral cortex yet all 10 subjects had uniformly low to absent tracer localization to the cerebellar cortex; significantly high tracer activity was noted within the subcortical white matter of the cerebellum in a symmetric manner in all cases. In consideration of studies that have shown amyloid deposits within the cerebellar cortex in $90 \%$ of pathologically proven cases of Alzheimer's disease, these findings raise questions about the actual clinical value of florbetapir PET imaging in evaluating cerebellar involvement and raises questions whether PET imaging of this tracer accurately portrays patterns of amyloid deposition, as there is rapid hepatic metabolism of the parent compound after intravenous injection. Possible links to Alzheimer's disease related alterations in blood-brain barrier permeability to the parent compound and subsequent radiolabelled metabolites are discussed as potential mechanisms that could explain the associated localization of the tracer to the brainstem and subcortical white matter within the cerebrum and cerebellum of Alzheimer's disease patients.
\end{abstract}

\section{Introduction}

Alzheimer's disease is a devastating health problem of an increasing magnitude within the expanding aging population that requires definitive diagnostic methods. In this regard, 18F-FDG PET imaging has been proven to be very helpful in identifying areas of focal posterior parietal hypometabolism linked to Alzheimer's disease (AD); other PET imaging methods include 11C-flumazenil that reveals no loss of benzodiazepine receptors and hence preservation of the GABAergic neuron population within dysfunctional posterior parietal cortical regions of Alzheimer disease patients. 1,2

Much interest has been generated on the use of $18 \mathrm{~F}$ and $11 \mathrm{C}$ labeled compounds that bind to amyloid plaque on postmortem brain tissue sections, but generate conflicting data with regards to their in-vivo distribution after intravenous injection to living subjects. Detailed neuropathologic studies over many years have consistently shown amyloid deposition within cortical regions in a non-homogenous distribution with predominance of involvement within the temporal lobes; however the new PET agents that display amyloid binding in-vitro display surprisingly high levels of activity throughout the white matter in a confluent somewhat symmetric manner in vivo - for those affected with $\mathrm{AD}$, there is additional penetration of tracer into the cerebral cortex and does not display the anticipated regional differences (no obvious proclivity for the hippocampus and temporal lobe structures). Furthermore, cerebral retention of the $18 \mathrm{~F}$ flubetapir tracer is not convincingly demonstrated on time activity curves after IV injection with patterns showing a spike after injection with subsequent wash out from cerebral tissues. ${ }^{3,4}$ As there is rapid metabolism of the tracer with only $35 \%$ of unchanged parent remaining at 10 minutes, ${ }^{3}$ it was suspected that tracer localization of the brain may depend on other factors such as Alzheimer related alterations in blood brain barrier permeability to the labeled metabolites. Since amyloid plaques are well described in the cerebellum of Alzheimer patients, ${ }^{5}$ further investigation was carried out here to understand why there is essentially absent flubetapir tracer activity within the cerebellar cortex.

\section{Materials and Methods}

Participants for the IDEAS trial signed informed consent forms approved by the institutional review board. Imaging of intravenously administered $18 \mathrm{~F}$-flubetapir ( 5 to $10 \mathrm{mCi}$ ) was accomplished per the standard protocol followed of the IDEAS trial with PET imaging commencing 30 minutes after injection and lasting 15 minutes with CT attenuation correction applied. All participants had vitamin B12 and TSH measured prior to entry, and all had brain CT or MRI within 12 months of the study. Although not required by the IDEAS trial, all patients described here had brain SPECT or PET imaging of the brain as well of cerebral perfusion or glucose metabolism respectively. Ages for the study group of 10 participants
Correspondence: Michael A. Meyer, Department of Neurology, Guthrie Clinic, 130 Centerway, Corning, NY 14830, USA.

Tel.: +1.607.973.8000.

E-mail: michaelandrewmeyer@gmail.com

Key words: PET; florbetapir; amyloid; cerebellum; Alzheimer's disease.

Funding: we acknowledge the contributions of the IDEAS Study and its collaborators (www.ideas-study.org)

Acknowledgements: The authors wish to thank Jon-Kar Zubieta, MD, PhD

Stimson Presidential Endowed Chair of Psychiatry, University of Utah for reviewing the manuscript.

Received for publication: 2 March 2018 Accepted for publication: 18 June 2018.

This work is licensed under a Creative Commons Attribution NonCommercial 4.0 License (CC BY-NC 4.0).

CCopyright M.A. Meyer et al., 2018 Licensee PAGEPress, Italy

Neurology International 2018; 10:7666

doi:10.4081/ni.2018.7666

described here ranged from 68 to 85 years (mean age: 75.5 years); disease severity ranged form mild to moderate to severe for suspected Alzheimer's disease. No adverse events were observed; all patients were reevaluated at the 90-day mark after PET. Reconstructed images in the sagittal and coronal planes through the cerebellum were selected for composite review and comparison.

\section{Results}

As shown in figure one, adequate quality PET images were obtained in all ten cases without significant motion artifact. Only one of ten cases had lack of penetration of tracer into the cerebral cortex, consistent with a pattern thought to be inconsistent with Alzheimer's disease. This 70 year old patient (case number 10; Figures 1 and 2A) had unusual encephalopathic behavior and squinting of her eyes with memory loss that later improved with resolution of ocular abnormalities. A typical Alzheimer case is shown in Figure 1 (case 1) and Figure 2B revealing diffuse penetration of tracer into the cortical regions; the patient is a 78 year old with moderately severe memory loss attributable to late onset Alzheimer's disease with a left posterior parietal perfusion defect noted on SPECT. However, in both 
of these two cases, as well for all the other 8 cases, there was little to no tracer activity evident in the cerebellar cortex (Figure 1). Although the subcortical white matter displayed significant activity within the subcortical white matter of both cerebellar hemispheres, little to no activity was found in the cerebellar cortex. Surprisingly intense activity was consistently seen within the brain stem (see cases 5,7,9,10 with case 9 showing more intense activity in the brain stem and cerebellar subcortical white matter than the occipital and parietal cerebral cortex), which is seemingly inconsistent with the known patterns of amyloid deposition in Alzheimer's disease. As prior pathologic studies have shown a gradient of A4 beta amyloid activity in $\mathrm{AD}$, where more plaques were seen in the midbrain and least in the medulla, ${ }^{6,7}$ with healthy controls showing little to no brain stem plaque, the PET scan findings become hard to explain; as explained below, it is hypothesized that 18F-flubetapir metabolites with no specific avidity for amyloid could contribute significantly to the image and appear in the brain stem, related to possible alterations in the blood-brain barrier but this is only hypothetical and not proven in any way.

\section{Discussion}

Although the parent compound has been shown to bind to amyloid plaques within postmortem tissue, its' distribution in-vivo after intravenous injection is likely variable due to rapid metabolism with possible variable permeability of the labeled metabolites in being able to cross the bloodbrain barrier. However, recent studies have shown that the parent compound is rapidly metabolized with only $35 \%$ of unchanged parent remaining at 10 minutes. ${ }^{3}$ Dynamic whole body imaging at sequential time points after injection document rapid hepatic metabolism, with very little activity left in the brain by 30 minutes later with fairly intense activity passing from the liver into the gallbladder at that time point; ${ }^{6}$ highest radiation absorbed dose were the gallbladder, upper large intestine, small intestine, liver, and urinary bladder at 143, 75, 66, 64, and $27 \mu \mathrm{Sv} / \mathrm{MBq}$, respectively. Appreciable minor bladder activity becomes evident by 186 minutes post injection. Time activity curves demonstrate an initial spike followed by rapid wash out from the cerebellar and cerebral cortex; 4 there appears to be no pattern for extraction and retention by the brain with no pattern for increasing activity over time - only steady washout is noted, much of which likely consists of radiolabelled hepatic metabolites that may possibly have the ability to cross the cortical blood-brain barrier of Alzheimer patients but not in normal individuals as one possible yet unproven explanation of the imaging data.
The lack of tracer activity in the cerebellum is of concern as multiple pathologic studies confirm the presence of amyloid plaques in Alzheimer disease. Braak and

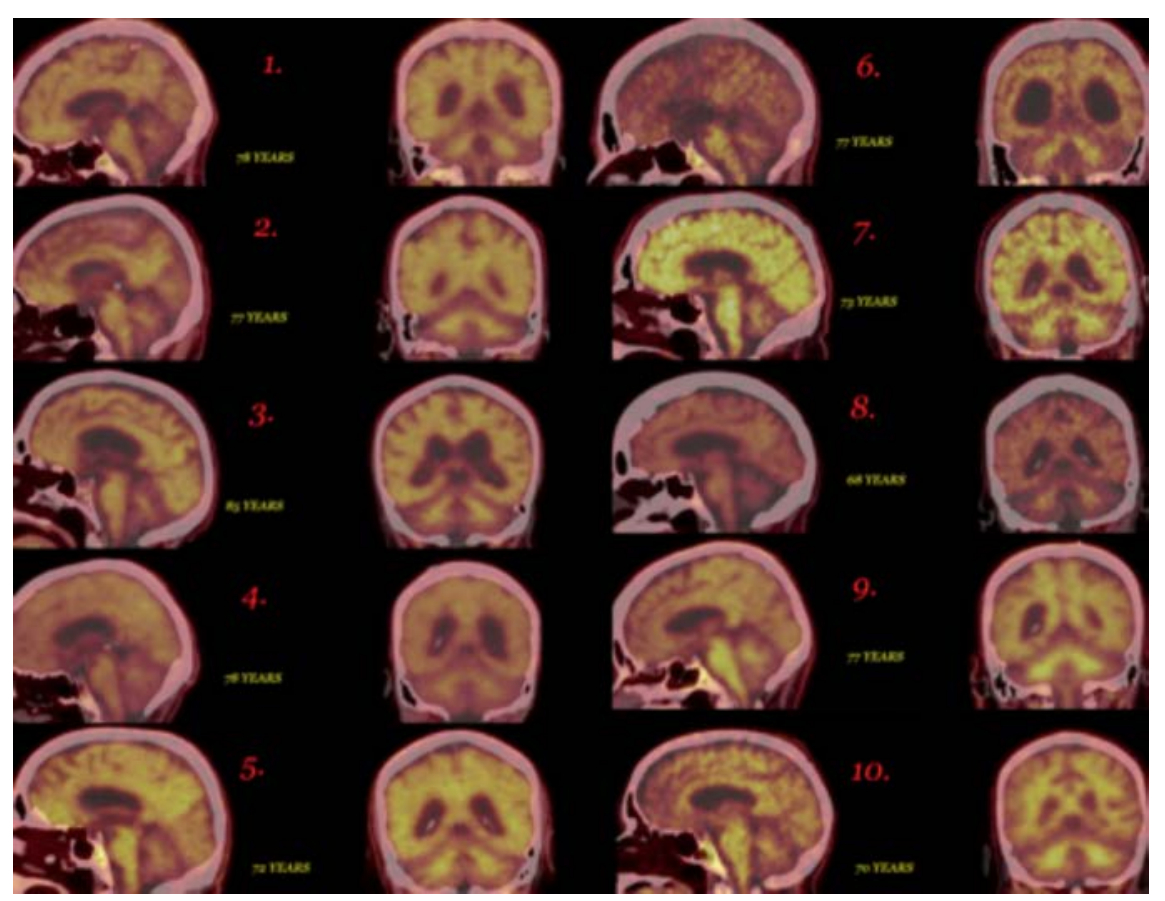

Figure 1. Composite analysis of all ten patients displaying 18F-flubetapir in the sagittal and coronal planes.

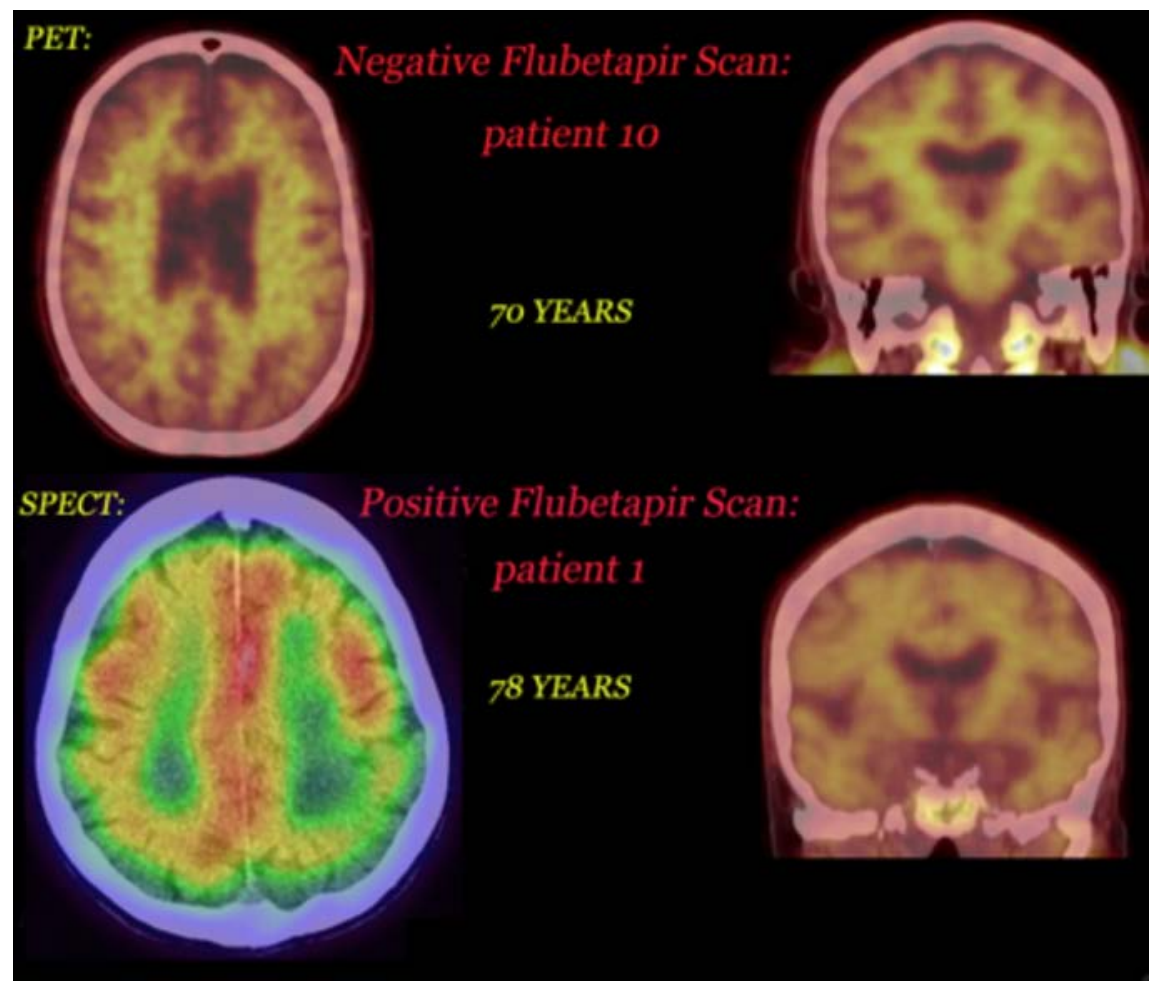

Figure 2. PET images depicting negative results (top) and positive results consistent with Alzheimer's disease (bottom). 
colleagues found large numbers of amyloid plaques in the cerebellar cortex of demented individuals but not in controls. Most of the plaques were seen in the molecular layer8 and were large and extended from the upper surface down to the Purkinje cell layer. Yamaguchi et al. described beta amyloid plaques in the molecular layer of the cerebellum. ${ }^{9}$ Mann et al. studied the cerebellum of Down's syndrome cases and concluded that: ...diffuse plaques of the cerebellum and striatum contain only A beta 42(43) and that, in contrast to those of the cerebral cortex, these do not 'mature' during their lifetime into A beta 40 containing plaques. 10 Barcikowska et al. studied brain tissue from 100 stroke patients older than 80 years, of whom $18 \%$ had dementia and found positive amyloid immunoreactivity in the cerebrum in 71 of 100 cases; cerebella of 31 subjects of 71 with cerebral amyloidosis also revealed amyloid deposits. ${ }^{11}$

Wegiel and colleagues 12 carefully studied cerebellar morphometrics and amyloid plaque morphology in severe final stages of Alzheimer's and concluded that significant atrophy was found with a decrease in the volume of the molecular layer by $24 \%$ and a decrease of the granular layer by $22 \%$ in comparison with controls. The $32 \%$ decrease in the total number of Purkinje neurons were found along with a $30 \%$ reduction in the number of granule cells They concluded that the ...correlation between the temporal duration of $A D$ and both the decrease of the total number of granule cells $(\mathrm{r}=0.86, \mathrm{P}<0.01)$ and the volumetric loss of the molecular $(\mathrm{r}=0.73$, $\mathrm{P}<0.05)$ and granular $(\mathrm{r}=0.93, \mathrm{P}<0.001)$ layers of the cerebellar cortex indicates that these cerebellar atrophic changes are likely to be related to the basic pathologic process of AD. Similarly, the correlation between the most complex parameter the atrophy of the cerebellar cortex and the Functional Assessment Staging (FAST) measure of the clinical severity of $A D$ at the time of demise $(\mathrm{r}=0.63, \mathrm{P}<0.05)$ as well as with the duration of $A D(\mathrm{r}=0.78, \mathrm{P}<0.01)$ indicates that cerebellar pathology, when viewed holistically, evolves continuously in association with clinical changes throughout the clinically manifest course of $A D$.

Selkoe and colleagues found that diffuse cerebellar plaques were detected in 47 of 100 cases of clinically and pathologically diagnosed $\mathrm{AD}$ and in none of 40 control subjects. ${ }^{13}$ The 1993 study of Cole and colleagues ${ }^{14}$ revealed an important age effect: cerebellar amyloid plaques were found in $93 \%$ in those who developed dementia before 65 years of age; only $56 \%$ cases were positive when dementia started after 75 years. They also studied brain stem involvement and noted that the severity of cerebellar changes corresponded to the involvement of the brain stem nuclei that are known to interconnect with the cerebellar cortex.

\section{Conclusions}

In summary, it is clear that the cerebellum is significantly affected in Alzheimer's disease. A $12.7 \%$ reduction in cerebellar volume has been noted 15 in severely affected Alzheimer disease patients analyzed postmortem. For reasons that are not yet clear, in-vivo study of $18 \mathrm{~F}$-Flubetapir by PET fails to reveal any significant activity within the cerebellar cortex of Alzheimer disease patients studied here, raising questions regarding the specificity of the tracer. Rapid hepatic metabolism of the tracer has been discussed here, raising questions regarding the likelihood that radiolabelled metabolites with no specific ability to bind amyloid may heavily contribute to the activity imaged at the 30 to 45 minute time interval after injection; the possibility of altered blood-brain barrier permeability to the parent compound and subsequent metabolites need to be considered here as well. Overall, it is concluded that $18 \mathrm{~F}$-florbetapir PET imaging was insensitive to detecting cerebellar amyloid deposits in this relatively small study of 10 patients; this stands in contrast to the fact that significantly numerous cerebellar amyloid plaques have been found by numerous postmortem neuropathologic studies. More research is clearly needed.

\section{References}

1. Mann DM, Jones D, Prinja D, Purkiss MS. The prevalence of amyloid (A4) protein deposits within the cerebral and cerebellar cortex in Down's syndrome and Alzheimer's disease. Acta Neuropathol 1990;80:318-27.

2. Meyer M, Koeppe RA, Frey KA, et al. Positron emission tomography measures of benzodiazepine binding in Alzheimer's disease. Arch Neurol 1995;52:314-7.

3. Ottoy J, Verhaeghe J, Niemantsverdriet $\mathrm{E}$, et al. Validation of the Semiquantitative Static SUVR Method for 18F-AV45 PET by Pharmacokinetic Modeling with an Arterial Input
Function. J Nucl Med 2017;58:1483-9.

4. Wong DF, Rosenberg PB, Zhou Y, et al. In vivo imaging of amyloid deposition in Alzheimer disease using the radioligand 18F-AV-45 (florbetapir [corrected] F 18). J Nucl Med 2010;51:913-20.

5. Mann DM, Jones D, Prinja D, Purkiss MS. The prevalence of amyloid (A4) protein deposits within the cerebral and cerebellar cortex in Down's syndrome and Alzheimer's disease. Acta Neuropathol 1990;80:318-2.

6. Joshi AD, Pontecorvo MJ, Adler L, et al. Radiation dosimetry of florbetapir $\mathrm{F}$ 18. EJNMMI Res 2014;4:4.

7. Brilliant M, Elble RJ, Ghobrial M, Struble RG. Distribution of amyloid in the brainstem of patients with Alzheimer disease. Neurosci Lett 1992;148:23-6.

8. Braak H, Braak E, Bohl J, Lang W. Alzheimer's disease: amyloid plaques in the cerebellum. J Neurol Sci 1989;93:277-87.

9. Yamaguchi H, Hirai S, Morimatsu M, et al. Diffuse type of senile plaques in the cerebellum of Alzheimer-type dementia demonstrated by beta protein immunostain. Acta Neuropathol 1989;77:314-9.

10 Mann DM, Iwatsubo T. Diffuse plaques in the cerebellum and corpus striatum in Down's syndrome contain amyloid beta protein (A beta) only in the form of A beta 42(43). Neurodegeneration 1996;5:115-20.

11. Barcikowska M, Kujawa M, Wiśniewski H. beta-Amyloid deposits within the cerebellum of persons older than 80 years of age. Neuropatol Pol 1992;30:285-93.

12. Wegiel J, Wisniewski HM, Dziewiatkowski J, et al. Cerebellar atrophy in Alzheimer's disease-clinicopathological correlations. Brain Res 1999;818:41-50.

13. Joachim CL, Morris JH, Selkoe DJ. Diffuse senile plaques occur commonly in the cerebellum in Alzheimer's disease. Am J Pathol 1989;135:309-19.

14. Cole G, Neal JW, Singhrao SK, et al. The distribution of amyloid plaques in the cerebellum and brain stem in Down's syndrome and Alzheimer's disease: a light microscopical analysis. Acta Neuropathol 1993;85:542-52.

15. Andersen K, Andersen BB, Pakkenberg B. Stereological quantification of the cerebellum in patients with Alzheimer's disease. Neurobiol Aging 2012;33:197.e11-20. 